\title{
Quaderni
}

QUADERNI Communication, technologies, pouvoir

92 | Hiver 2016-2017

Les artistes à l'école : fin d'une illusion ou utopie en devenir?

\section{Jean-Michel Eymeri-Douzans, Xavier Bioy et Stéphane Mouton (dir.), Le Règne des entourages. Cabinets et conseillers de l'exécutif}

\section{Laurent Godmer}

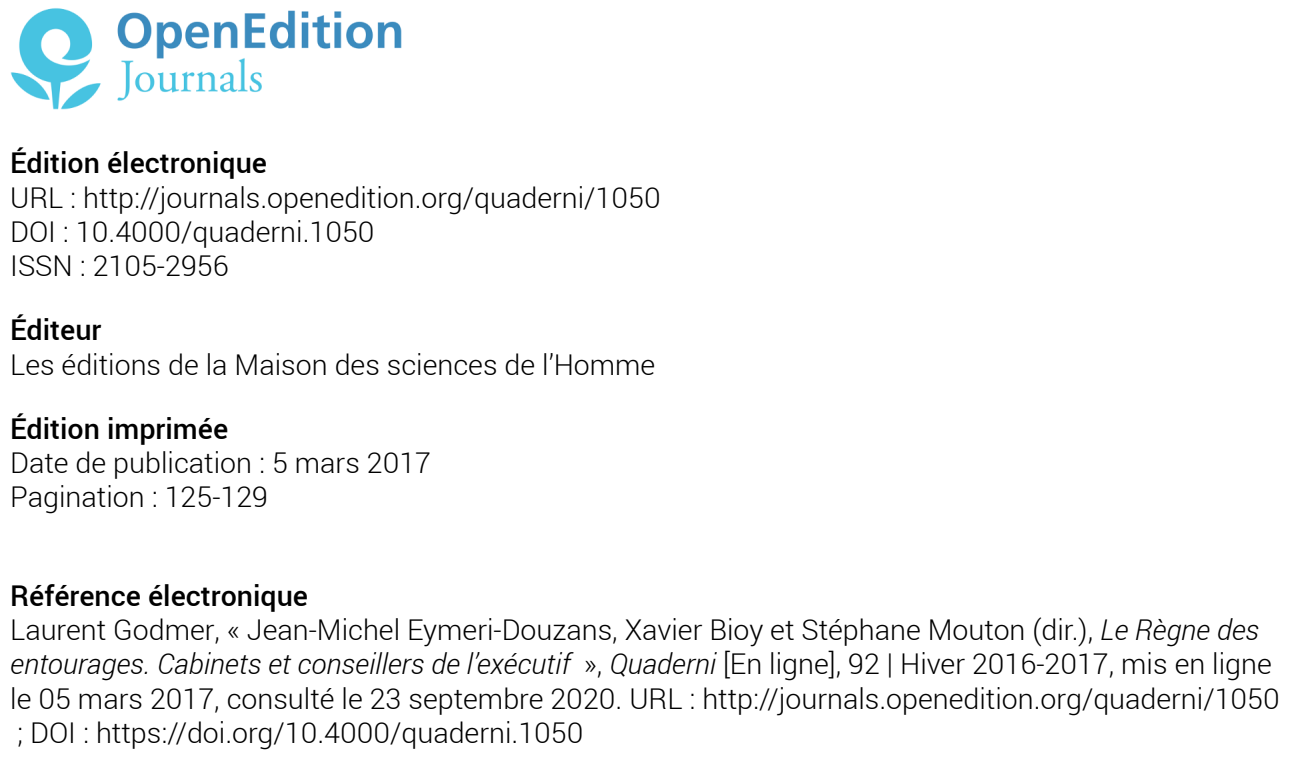

Tous droits réservés 


\section{Compte rendu}

Le Règne des entourages.

Cabinets et conseillers de l'exécutif

Jean-Michel Eymeri-Douzans, Xavier Bioy

et Stéphane Mouton (dir.)

Presses de Sciences Po, coll. "Académique", Paris, 2015

par Laurent Godmer

Université Paris-Est

Qu'est-ce que gouverner? Qui sont ceux qui font fonctionner au quotidien la machinerie gouvernementale? Autant d'interrogations auxquelles tâche notamment de répondre Le Règne des entourages.

Le travail gouvernemental repose de plus en plus sur des équipes de conseillers. Comme l'indiquent dans une longue introduction (94 pages) Jean-Michel Eymeri-Douzans et Xavier Bioy, «nos régimes politiques sont devenus des "républiques de conseillers" dont le procès est partout instruit de façon rituelle » (p. 17). La somme qu'ils ont codirigée avec Stéphane Mouton vise à explorer une institution, le cabinet de l'exécutif (cabinets ministériels, des présidents, des chefs de gouvernement), qui joue un rôle majeur dans les rouages du pouvoir. Elle est centrée sur la France, avec quelques contrepoints ouest-européens et nord-américains.

Cet ouvrage très riche décrit des entourages au volume croissant (« la comparaison entre 1959 et 2014 fait ressortir une croissance de $75 \%$ (effectifs officiels), voire de $80 \%$ (officieux inclus) de la taille des cabinets » (p. 31)), un processus international que l'on pourrait nommer « cabinetisation » du monde politique. Le grand mérite de ce livre est de lever un coin de voile sur ces curieux espaces curiaux que sont les cabinets. Leur prospérité est à relier à une technicisation de l'action gouvernementale, à la présidentialisation du pouvoir en général, et à la professionnalisation de l'activité politique en particulier. Les conseillers pallient le manque de temps des gouvernants dans un univers surmédiatisé, et participent à la gestion de la complexité 
de l'action publique : "L'ampleur, la diversité et la complexité des tâches sont devenues telles que nul ne gouverne plus seul. » (p. 48) Le travail de production de la décision publique nationale semble ainsi, comme ce qui relève du travail politique en général ${ }^{1}$, être de plus en plus collectivisé et chronophage. La grande force de cet ouvrage qui fait le point sur les « métiers d'entourage » (p. 73) est de documenter statistiquement la montée en puissance des conseillers des membres de l'exécutif français, en particulier sous la Cinquième République. Leur « pouvoir » est en effet grandissant, à tel point qu'est forgée l'hypothèse d'une « mainmise des collaborateurs sur le travail législatif $\gg(\text { p. 89) })^{2}$.

Si la construction de la monarchie a contribué à institutionnaliser des « conseils » (ch. 1) dans lesquels agissaient des auxiliaires du monarque, Le Règne des entourages s'attache à retracer sous la Révolution et surtout sous la Restauration (ch. 2), les origines davantage directes des cabinets ministériels. Ils étaient très réduits au début du XIX ${ }^{\mathrm{e}}$ siècle. L'on constate la stabilisation du terme et de l'institution « cabinet » sous la Restauration et pour tous les ministères sous la monarchie de Juillet (ch. 3). S'il connaît des formes de formalisation de son organisation et de ses activités sous le Second Empire (ch. 4), « le cabinet ministériel se bureaucratise et se structure sous la Troisième République pour devenir une institution centrale de tout ministère » (Gildas Tanguy, ch. 5, p. 238). Cette généalogie montre la lenteur de son institutionnalisation, ses évolutions, mais aussi son enracinement croissant dans les couloirs du pouvoir, puisqu'il apparaît alors comme le « lieu où se forge la décision politique » (p. 239). Le cabinet est un espace de plus en plus peuplé et hiérarchisé sous la Quatrième République (le régime de Vichy est rapidement évoqué (ch. 6, p. 244-245)). Après la Seconde guerre mondiale, c'est surtout la féminisation de la population des conseillers et la présence importante des anciens élèves de l'École nationale d'administration (ENA) qui sont marquantes. Malgré la rationalisation du recrutement et des fonctions des cabinets, la catégorie « entourages » n'échappe pas à un certain flou : des conseillers demeurent « officieux »; "on peut faire partie de l'entourage sans appartenir au cabinet (voir la figure du "visiteur du soir") » (Jacques Gerstlé, ch. 22, p. 646) ; enfin, les amis, les figures du parti politique et de la famille peuvent aussi être consultés par un ministre, comme en Allemagne (ch. 28).

Cette relative instabilité « statutaire » des conseillers des exécutifs contraste avec une forte «stabilité » sur le plan sociodémographique. Plusieurs
1. Cf. Didier Demazière et Patrick Le Lidec (dir.), Les mondes du travail politique. Les élus et leurs entourages, Rennes, Presses universitaires de Rennes, 2014; Laurent Godmer et Guillaume Marrel,

La politique au quotidien. L'agenda et l'emploi du temps d'une femme politique, Lyon, ENS Éditions, 2015.

2. Comme le suggère par exemple un roman paru également en 2015 dont le principal protagoniste est directeur de cabinet: « En réalité c'était plutôt le directeur de cabinet qui avait un pouvoir de vie ou de mort sur le ministre. II pouvait l'asphyxier en le laissant crouler sous les arbitrages les plus anecdotiques, l'ensevelir sous ces parapheurs bordeaux en cuir fatigué qu'il fallait examiner tous les soirs, ou au contraire lui cacher l'essentiel en barrant le chemin aux informations les plus sensibles, pour se laisser le privilège de décider lui-même. Il était potentiellement le vrai maître $d u$ ministère. » Thomas Bronnec, Les Initiés, Paris, Gallimard, coll. "Série noire ", 2015, pp. 50-51. 
contributions administrent la preuve de la « permanence des soubassements sociaux des cabinets ministériels » (Sylvain Laurens, ch. 12, p. 406). Les analyses prosopographiques permettent de relativiser les «idées reçues » : elles objectivent le fait que les conseillers sont rarement des intimes du ministre et mettent au jour l'existence d'un processus de fonctionnarisation de leur recrutement, caractérisé par « la faiblesse des écarts entre droite et gauche » (Thomas Alam, Igor Martinache et Jérémie Nollet, ch. 14, p. 436 et 441). Si la proportion de fonctionnaires dépasse la moitié des membres des cabinets ministériels (souvent environ près des deux tiers sont des « technos »), celle des anciens élèves de l'ENA régresse parfois, mais cette baisse est « compensée » par le fait que, sous les présidences Chirac et Sarkozy, plus des deux tiers des directeurs et directeurs adjoints de cabinets sont passés par cette école, ce qui illustre la hiérarchisation de ces institutions. Ces dernières fonctions étaient d'ailleurs alors occupées quasi uniquement par des hommes, alors que les cabinets ont connu une féminisation accentuée au début du XXI ${ }^{\mathrm{e}}$ siècle (un quart des membres) (id., p. 446) : « Les collaborateurs ministériels demeurent très largement issus d'une élite, masculine, parisienne, d'origine sociale favorisée et fortement diplômée, avec une part toujours importante d'énarques. » (id., p. 454) La domination de ces derniers est encore plus nette dans l'entourage du président Hollande (ch. 15, p. 468). L'expérience de membre de cabinet est un capital qui se révèle fréquemment un accélérateur de carrière, essentiellement dans la fonction publique (ch. 16), mais aussi parfois dans le champ politique (ch. 17). Les cabinets jouent un rôle crucial, bien qu'ils soient (plus ou moins) contraints par une autre hiérarchie, celle qui subordonne ceux des ministres à ceux des chefs de l'État et du gouvernement (ch. 21). Cet ouvrage passionnant décrit surtout un modèle « français » de fonctionnement des entourages, axé sur le «modèle du portefeuille et la tutelle des services » (Michel Mangenot, ch. 24, p. 690), mais sans collégialité : les membres d'un cabinet traitent de leur secteur, au contraire de ceux des commissaires européens par exemple.

Sous-codifié sur le plan juridique (ch. 8, 9 et 10), le travail de ces entourages de l'exécutif est, à rebours d'une doxa arguant de leur technocratisation, avant tout politique : «La fonction de collaborateur au cabinet [...] peut être caractérisée comme l'usage de compétences techniques mises au service d'une politique » (Françoise Dreyfus, ch. 7, p. 280). En ce sens, ce livre fait œuvre utile, qui montre que les dimensions « techniques » du travail politique (relatives aux politiques publiques (policies)) et ses dimensions « politiques » 
(relatives à « la » politique (politics)) sont fortement imbriquées. De surcroît, il évoque, autant pour les États-Unis d'Amérique (ch. 29) que pour la France ou plusieurs États ouest-européens, l'importance des activités de communication dans le travail des entourages. L'omniprésence des cabinets dans l'élaboration des politiques gouvernementales semble être en tous cas aujourd'hui nettement confirmée (ch. 19). Le Règne des entourages procède à une démystification de ces lieux de pouvoir parfois construits comme mystérieux. Il éclaire sur des évolutions majeures de l'art de gouverner, désormais davantage collectif (ch. 18), davantage technique, mais qui demeure très « politique » derrière cette façade.

Le Règne des entourages mériterait indubitablement un prolongement davantage international. Les auteurs de l'introduction indiquent ne "pouvoir conduire ici une démonstration aboutie qui requerrait une vaste enquête internationale sur les entourages de l'exécutif, qui reste à faire et sera peut-être entreprise » (p. 29). Par ailleurs, il serait utile de regarder le fonctionnement des entourages des grands exécutifs territoriaux (surtout ceux des grandes villes et des grandes régions) qui sont peu décortiqués, sauf par Yves Pourcher (ch. 23). Cette étude du fonctionnement d'un modèle national qui fait référence ne conduit-elle pas parfois à survaloriser le pouvoir des conseillers ? Les entourages français apparaissent indéniablement plus « forts » par exemple que ceux de l'Italie soumis à une instabilité politique chronique (ch. 26) et bien moins critiqués pour leur amateurisme que leurs équivalents belges (ch. 25). Mais tout se passe comme si émergeait une contradiction potentielle entre une « république des entourages » censément puissante en France et les procès en indécision liés à des évolutions contextuelles (européanisation, décentralisation, médiatisation, instabilité, « crises »).

La solidité sociographique incontestable des analyses gagnerait également à être prolongée par des monographies relevant de démarches davantage ethnographiques et/ou biographiques (portant sur des membres de cabinets, sur le cabinet d'un ministère, sur le suivi d'une politique publique). Peu d'études décryptent la trajectoire de conseillers précis et, à l'exception du chapitre consacré au Royaume-Uni (ch. 27), l'ouvrage cite peu de conseillers ayant eu un rôle objectivable dans l'élaboration d'une politique. Le travail des membres des cabinets est fondé sur l' « interministériel » (ch. 20), qui est rythmé par les quelque deux mille réunions interministérielles annuelles et par un quotidien fait de la rédaction de notes (ch. 11, p. 374). Leur activité 
principale est « ce travail quotidien par lequel les membres des cabinets participent aux processus d'arbitrage constitutifs de la décision gouvernementale » (introduction, p. 87). Le développement de l'ethnographie du travail gouvernemental quotidien est une des conditions de possibilité d'une saisie affinée de l'univers des pratiques politiques et de la surdétermination supposée de la politique par la communication. Afin de continuer cette anatomie du travail de conseil politique, aborder encore davantage le « que font-ils ? » est d'un intérêt heuristique certain, qui peut passer par exemple par l'objectivation des emplois du temps des conseillers pour voir (ce) qui se cache derrière les « agendas de ministre ». 
